\title{
Influence of aquifer natural convection on deep borehole heat exchanger efficiency
}

\author{
Asal Bidarmaghz, ${ }^{1, *}$ and Guillermo A. Narsilio ${ }^{2}$ \\ ${ }^{1}$ School of Civil and Environmental Engineering, University of New South Wales, Sydney, Australia \\ ${ }^{2}$ The University of Melbourne, Department of Infrastructure Engineering, Engineering Block B 208, Parkville, VIC 3010, Australia
}

\section{Introduction}

Deep borehole heat exchangers with lengths on the order of several kilometres can be utilised for geothermal heat extraction using deep borehole heat exchangers (BHEs), e.g., closed U-loop or coaxial borehole heat exchangers (CBHEs). The thermal yield of such systems has been investigated through computational simulations, analytical analysis, and full-scale experiments. Despite the recent knowledge advancement in deep geothermal extraction via BHEs, the existing studies mainly involve conductive heat transfer in the ground, neglecting the effects of groundwater and heat convection (natural or forced) within the aquifer on the thermal efficiency of these systems. Considering the fluctuations in groundwater density at high temperatures, the role of natural convection in deep saturated formations could be crucial in evaluating the ultimate thermal yield of deep BHEs. To test this hypothesis, in this study, a 3D finite element model is developed to capture heat and mass transport in non-homogeneous ground encompassing a 5 $\mathrm{km}$ coaxial borehole heat exchanger. In addition to the coupled heat transfer and fluid flow in the ground and the carrier fluid in the CBHEs, the model accounts for natural convection in deep saturated formations - a widely overlooked phenomenon in heat transfer mechanisms in porous media. The ultimate goal of this study is to evaluate the extent to which the thermal performance of deep BHEs is affected by natural convection, which encompasses accounting for groundwater parameters' temperature dependency, the resultant buoyancy in groundwater and the effects of gravity. To identify scenarios in which natural heat convection might play a critical role in deep CBHEs, parametric studies are undertaken for various ranges of permeability, carrier fluid velocity and CBHEs depth.

\section{Heat and mass transport modelling in and around deep coaxial borehole heat exchangers}

To capture the effects of density-driven groundwater flow and natural convection on the heat transfer process and the consequent temperature distribution in the aquifer, several inter-dependencies are prescribed: i) the groundwater flow is coupled to the heat transfer occurring in the aquifer via the Darcy velocity, ii) groundwater density in the aquifer is temperature dependent, iii) the CBHE's wall and carrier fluid temperatures are coupled to the aquifer and the impervious layers' temperatures. Figure 1 illustrates the system's geometry and set-up to investigate the heat transfer processes around and inside up to 4,650 m deep CBHEs.

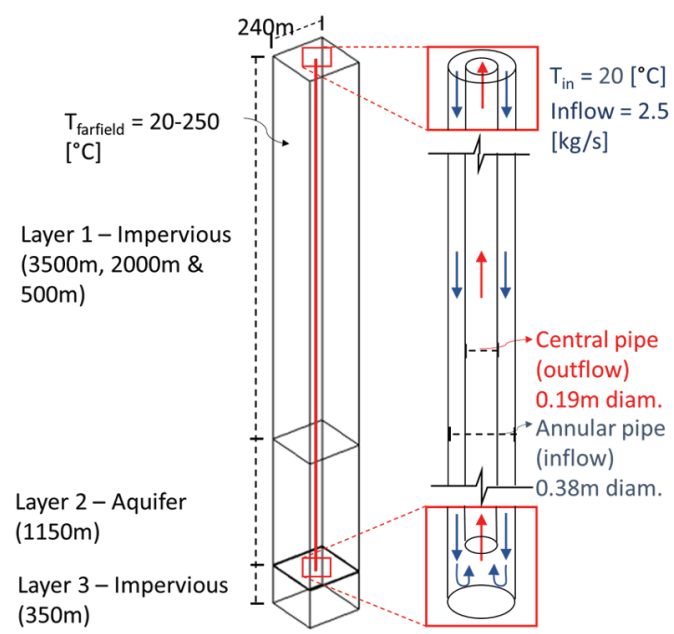

Fig. 1: Deep CBHEs setup in a layered deposit.

In CBHEs, heat delivery is achieved via a central coaxial outflow pipe and the carrier fluid inflow via an annular pipe (see Figure 1-right). The - up to $-5 \mathrm{~km}$ deep model encompasses four different domains: i) the ground, ii) the fully saturated formation (aquifer), iii) the coaxial borehole, and iv) the carrier fluid. The aquifer $(1,150 \mathrm{~m}$ in depth) sits between two relatively impervious layers,

\footnotetext{
* Corresponding author: a.bidarmaghz@unsw.edu.au
} 
$3,500 \mathrm{~m}$ and $350 \mathrm{~m}$ deep, respectively. With $0.38 \mathrm{~m}$ outer and $0.19 \mathrm{~m}$ inner diameters, the coaxial borehole heat exchanger extends up to $4,650 \mathrm{~m}$ below the surface. The inner pipe wall (central pipe) is $0.03 \mathrm{~m}$ thick. Thermal continuity is considered as the boundary between the CBHE's annulus and the ground. The carrier fluid circulating inside the coaxial boreholes is modelled as a turbulent incompressible flow (Wilcox, 1998). The Reynolds-averaged Navier-Stokes (RANS) equations are implemented for conservation of momentum, as well as the continuity equation for conservation of mass. Heat exchange within the CBHE, carrier fluid, the surrounding ground and groundwater are captured by coupling and solving the conductive and convective heat transfer equations with fluid flow in a porous medium (aquifer). Groundwater flow in a fully saturated formation is described by Darcy's Law (details can be found in Bidarmaghz and Narsilio, 2016). The 3D numerical model is developed and implemented in the finite element package COMSOL Multiphysics.

\section{Results and Discussion}

This work - for the first time - investigates the contribution of natural convection in deep aquifers to the thermal efficiency of deep coaxial borehole heat exchangers. The numerical model developed here is used to study the effects of the spatial variability of the ground, the aquifer and the carrier fluid temperatures resulting from the continuous heat extraction via CBHEs. Various scenarios of carrier fluid inflow rate, aquifer's intrinsic permeability $(\mathrm{k})$ and CBHE's depth are considered in this study to identify situations in which neglecting the natural convection in the aquifer might have a profound impact on the total thermal yield of CBHEs. It was concluded that increasing the inflow rate has a more significant impact on the thermal efficiency when the aquifer's permeability is high (Figure 2). This is mainly associated with the large variations in groundwater density, hence the resultant temperature gradient in highly permeable aquifers.

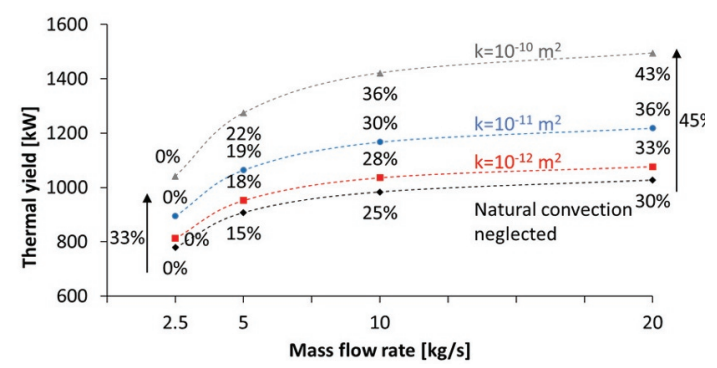

Fig. 2: Comparative illustration of 50-year thermal yield from a $5 \mathrm{~km}$-deep system.

As the portion of CBHE depth embedded in a permeable layer increases, the thermal performance of the CBHE has shown more sensitivity to the aquifer's permeability (e.g., the $2 \mathrm{~km}$ model), and it was observed from the results in Figure 3 that a CBHE installed in a highly permeable aquifer could have up to $57 \%(170 \mathrm{~kW}$ vs. $108.9 \mathrm{~kW}$ for a $2 \mathrm{~km} \mathrm{CBHE}$ ) higher thermal yield than a case in which permeability is low or natural convection is overlooked. This indicates that natural convection has a more pronounced effect on CBHE heat transfer s than the carrier fluid inflow rate in the $\mathrm{CBHE}$ as it engages more of the aquifer along its depth. This is in contrast with the behaviour of longer CBHEs and less relative engagement of the aquifer (e.g., $5 \mathrm{~km}$ model), in which the natural convection and carrier fluid inflow rate could have a similar effect on the system's efficiency ( $43 \%$ vs $45 \%$ increase in thermal yield, as shown in Figure 2).

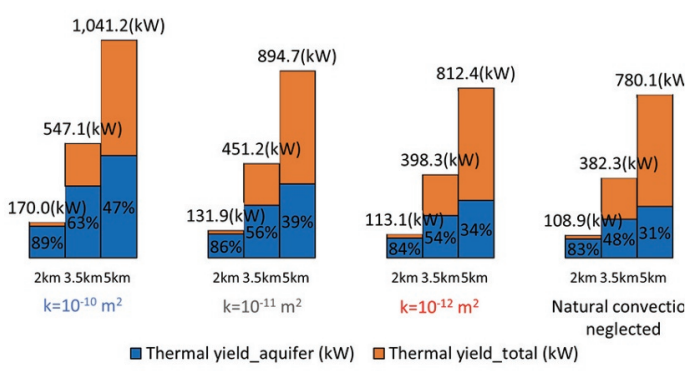

Fig. 3: Aquifer's contribution to CBHE's thermal yield.

\section{References}

1. Wilcox, D. C. 1998. Turbulence modeling for CFD, DCW industries La Canada, CA.

2. Bidarmaghz, A. \& Narsilio, G. A. 2016. Shallow Geothermal Energy: Emerging Convective Phenomena in Permeable Saturated Soils. Géotechnique Letters, 6, 119-123. 